\title{
Les banques islamiques sont-elles si islamiques que cela? \\ Conséquences en matière de fonds propres réglementaires
}

Mohammad Bitar*

Docteur en Finance

Philippe Madiès**C

\section{Professeur de Finance}

\section{Résumé}

En théorie, les banques islamiques sont des institutions qui appliquent le concept de partage de profits et de pertes (PPP). Néanmoins, la littérature montre que, dans la pratique, les banques islamiques reposent essentiellement sur des opérations commerciales (non-PPP) et marginalement sur les instruments PPP. Dans ce papier, nous démontrons que le type d'intermédiation privilégié par les banques islamiques n'est pas très différent de celui des banques conventionnelles. Tout d'abord, nous analysons les activités pratiquées par les banques islamiques à la lumière d'un modèle d'intermédiation pur et d'un modèle hybride. Ensuite, nous montrons que les banques islamiques pratiquent le concept de PPP au passif du bilan mais les opérations commerciales non-PPP à l'actif du bilan. Ainsi, le contrat Mourabaha représente à lui seul près de $80 \%$ du total des activités des banques islamiques. Enfin, nous démontrons à partir d'un échantillon de 656 banques dont 116 islamiques que le type d'intermédiation privilégié par les banques islamiques les rend plus capitalisées en matière de fonds propres réglementaires pondérés et non pondérés par le risque que leurs homologues conventionnelles.

\begin{abstract}
In theory, Islamic banks are profit-and-loss-sharing (PLS) institutions. Nevertheless, literature shows that, in practice, Islamic banks rely mainly on mark-up financing techniques compared to a marginal share of PLS instruments. In this paper, we demonstrate that Islamic banks intermediation model is not very different than conventional banks. First, we report pure and hybrid models to reflect the activities practiced by Islamic banks. Second, we show that Islamic banks use PLS arrangement at their liability side but mark-up financing techniques at their assets side. Islamic banks financing modes are driven by Mourabaha contract that represent almost $80 \%$ of their activities. Finally, we employ a sample of 656 banks - including 116 Islamic ones - to examine the impact of their preferred intermediation model and find that Islamic banks have higher capitalization in the form of risk-based and non-risk-based capital ratios than conventional counterparts.
\end{abstract}

Mots clés : Banques islamiques - Intermédiation bancaire - fonds propres réglementaires

Keywords: Islamic banks - Banking intermediation - capital requirements

* École de gestion John-Molson, Université Concordia, Montréal, Canada.

Email : mohammad.bitar@concordia.ca

**C Université Grenoble Alpes et CERAG (UMR 5820 du CNRS).

Auteur correspondant. Responsable du Master 2 Banque et Finance. Adresse professionnelle : IAE site de Valence, 51 rue B. de Laffemas 26000 Valence. Tel. 04754197 71. Fax +33 (0)4 75418844. philippe.madies@iae-grenoble.fr

Adresse personnelle : 19, allée Maurice Utrillo 26000 Valence. Tel : 0675174232.

\section{Introduction}


Bien que les institutions financières conformes à la Charia ne détiennent que 1,5\% des actifs du système bancaire international, elles sont en forte croissance (Abedifar, Molyneux et Tarazi, 2013 ; Beck, Demirgüç-Kunt et Merrouche, 2013). Ainsi, sur la période allant de 1998 à 2005, les banques islamiques ont vu leurs actifs croître de $110 \%$ contre $5 \%$ pour les banques conventionnelles (Khan, 2010). En outre, selon une étude du Cabinet Ernest and Young (2012), le montant des actifs des institutions financières islamiques devrait atteindre plus de 1800 milliards de dollars à la fin de 2013 et 3400 milliards de dollars en 2018. Cela peut s'expliquer par la rente pétrolière des pays du Golfe, une certaine volonté du monde musulman de se démarquer des pays occidentaux et enfin le souhait d'étendre les principes de la Charia à l'ensemble des activités économiques et donc à la finance. Aussi, ce développement de la finance islamique ${ }^{\mathrm{i}} \mathrm{s}^{\prime}$ est produit à un moment où le système financier international se voit contraint par des réglementations prudentielles plus rigoureuses en réponse à la crise financière de 2007-2008.

Cependant, malgré cette image attractive, les banques islamiques font face à de multiples difficultés qui risquent de les rendre plus vulnérables lors de futures crises financières. Hassan et Dridi (2010) ont évalué l'impact de la crise financière de 2007-2008 en comparant banques islamiques et banques conventionnelles et se sont interrogés sur le Business model respectif de ces deux types institutions. Ils montrent que les banques islamiques sont plus profitables sur les périodes d'avant et d'après crise mais que les deux types de banques ont des niveaux de profitabilité similaires en 2008-2009. Pendant la crise, les banques islamiques ont cependant témoigné d'un dynamisme plus fort en matière de financement de l'économie.

Pour autant, ces chercheurs insistent en premier lieu sur le fait que le système bancaire islamique manque d'harmonisation entre les pays en matière de réglementation. En outre, les banques islamiques se tournent vers des opérations commerciales (i.e. opérations adossées à des actifs) pour faire face à la compétition des banques conventionnelles. Ce dernier point avait déjà été évoqué par Errico et Farahbaksh (1998). Kraisicka et Panth (2012) ont des conclusions qui vont dans le même sens lorsqu'ils examinent le cas malaysien qui permet de comparer directement banques islamiques et banques conventionnelles. Ils concluent qu'il y a dans le temps une convergence des deux modèles qui passe par une réduction de l'utilisation par les banques islamiques d'opérations qui font leur spécificité (instruments de partage de profit et de pertes (PPP)). Ces auteurs suggèrent en conséquence que les banques islamiques devraient se soumettre aux mêmes réglementations que les banques conventionnelles. Cela devrait passer 
pour eux par une plus forte collaboration entre l'Islamic Financial Services Board (IFSB) et la Banque centrale de Malaisie. Ils soulignent en outre la nécessité d'harmoniser et donc de réduire significativement les interprétations de la loi islamique entre les différents comités de Charia institués au niveau de chaque banque islamique. Enfin, d'autres auteurs comme Khan (2010), Abedifar, Molyneux et Tarazi (2013), et Beck, Demirgüç-Kunt et Merrouche (2013) font le constat d'un écart significatif entre ce que la loi islamique prévoit du business model des banques islamiques et ce qui l'en est dans la réalité.

Dans ce contexte, notre article s'interroge sur la réalité des particularités des banques islamiques par rapport aux banques conventionnelles et leur véritable modèle d'intermédiation. Cela nous conduit notamment à étudier empiriquement leurs similitudes et différences en matière de fonds propres réglementaires.

L'article est structuré comme suit. En utilisant les données des rapports annuels de 115 banques islamiques obtenus de la base de données IBIS sur la période 2000-2011, la première section discute de la réalité des spécificités des banques islamiques en confrontant les données chiffrées aux deux modèles d'intermédiations prévus par la loi islamique. Ensuite, une deuxième section est consacrée à l'impact des nouvelles orientations des banques islamiques sur leur niveau de fonds propres réglementaires. En outre, cette section propose une étude économétrique, avec différentes mesures de fonds propres réglementaires, sur un échantillon de base de 656 banques (dont 116 banques sont islamiques) issues de la base de données Bankscope. L'objet de cette section est donc de tester empiriquement l'impact de l'intermédiation actuelle des banques islamiques sur leur fonds propres cela en comparaison avec les banques conventionnelles qui nous servent de «benchmark ».

\section{Les banques islamiques: quel type d'intermédiation?}

Théoriquement, les principes qui guident les activités des banques islamiques sont fort différents des ceux des banques conventionnelles. La Charia impose des contraintes sur les opérations des banques islamiques de nature différente à celles imposées par la réglementation prudentielle qui s'applique aux banques conventionnelles. Partant du fait que les banques islamiques sont des institutions dont l'objectif final est de maximiser la valeur humaine et sociale, elles ne partagent pas les mêmes risques que les banques conventionnelles. Effectivement, les outils de financement des banques islamiques sont différents de ceux des banques conventionnelles. D'ailleurs, la réglementation internationale de Bâle est construite 
pour des banques conventionnelles. Cela rend compliqué, voire impossible, l'application précise des nouvelles normes de capital, de liquidité et de levier contenues dans Bâle III aux banques islamiques sans tenir compte de leurs spécificités (Bitar et Madiès 2013).

Les banques islamiques sont censées utiliser des opérations fondées sur le principe du partage des profits et des pertes (PPP) à l'actif comme au passif de leur bilan et des opérations adossées à des actifs appelées encore opérations commerciales. Certains auteurs critiquent le fait qu'en pratique les banques islamiques utilisent principalement les opérations adossées à des actifs et rarement les opérations de partage de profits et de pertes pour développer leur activité. Les opérations commerciales non-PPP sont tolérées par la loi islamique mais les préceptes de la Charia et la raison d'être des banques islamiques se retrouvent dans les opérations de PPP. Ainsi, Beck, Demirgüç-Kunt et Merrouche (2013), parmi d'autres, expliquent que le contrat de Mourabaha, extrêmement utilisé par les banques islamiques, n'est qu'un contrat de crédit-bail des banques conventionnelles adapté aux banques islamiques. D'ailleurs, ces auteurs ne trouvent pas de grande différence de business model, d'efficience et de stabilité lorsqu'ils comparent banques islamiques et banques conventionnelles sur un échantillon de 24 pays.

La question qui se pose est alors de savoir si la pratique actuelle des banques islamiques ${ }^{\mathrm{ii}}$ est conforme aux préceptes de la loi islamique et ce qui fait réellement leurs spécificités par rapport aux banques conventionnelles. Dans cette perspective, nous définissons tout d'abord deux modèles d'intermédiation que les banques islamiques peuvent choisir car jugés comme conformes à la Charia. Ensuite, nous proposons un travail empirique permettant de juger du modèle d'intermédiation le plus utilisés dans la réalité. Pour cela, nous nous fondons sur les rapports annuels des 115 banques islamiques obtenus de la base de données IBIS (Islamic Banks and Financial Institutions Information) sur la période 2000-2011. Enfin, nous examinons l'impact de ce type d'intermédiation sur les fonds propres réglementaires de ces banques en comparaison avec les banques conventionnelles.

\section{Les différents modèles d'intermédiation des banques islamiques}

Les banques islamiques opèrent comme des banques commerciales et des banques d'investissement. Théoriquement, les activités de ces banques peuvent se présenter sous forme 
de deux modèles. Il s'agit d'un premier modèle purement conforme à la Charia dont les opérations sont basées sur le principe PPP et un deuxième modèle hybride dont les activités (non-PPP) sont proches de celles des banques commerciales (Sundararajan et Errico, 2002).

\section{Modèle pur et conforme aux préceptes de la loi islamique (finance participative)}

Dans ce modèle connu aussi sous le nom de «Moudharaba deux tiers », la banque joue le rôle de Moudharib avec les déposants et de Rab-El-Mal avec les investisseurs. Il s'agit du concept de Moudharib youdharib où la banque s'inscrit comme un intermédiaire financier en effectuant deux opérations en parallèle. En premier lieu, les déposants-investisseurs ( $R a b$ - ElMal) placent leur fonds à la banque (Moudharib). Il s'agit d'une opération de Moudharaba au Passif. Ensuite, à l'Actif, la banque investit les fonds dans des projets d'investissements conformes à la Charia. Elle joue le rôle de Rab-El-Mal tandis que l'entrepreneur devient Moudharib. Pour ce faire, la banque utilise des instruments fondés sur le partage des profits et des pertes (c'est-à-dire Moudharaba) avec ses clients qui deviennent désormais des partenaires. Il s'agit donc d'une seconde opération de Moudharaba qui a lieu désormais à l'Actif.

Ainsi, la banque et ses clients investisseurs peuvent participer conjointement au financement et à la gestion des projets (Rab-El-Mal et Moudharib) jugés conformes à la Charia, en appliquant le principe de Mousharaka à l'Actif.

Ce modèle d'intermédiation est privilégié par la loi islamique puisqu'il combine deux opérations de nature PPP à l'Actif et au Passif du bilan. Pour cette raison, ce modèle ne requiert aucune réserve obligatoire concernant les comptes d'investissements des clients. Par contre, si les comptes d'investissements se confondent avec dépôts à vue, toute perte liée à des opérations d'investissement nécessite la constitution de réserves obligatoires (El-Hawary, Grais et Iqbal, 2007). Ainsi, la pratique du PPP qui favorise l'utilisation des comptes d'investissements au Passif pour financer des projets à la base du Moudharaba ou Mousharaka à l'Actif renforce la conformité des opérations bancaires aux préceptes islamiques et conduit à une gestion des risques moins complexe. En effet, ce type d'intermédiation assure le partage des risques avec les clients entrepreneurs et les clients déposants, ce qui améliore d'ailleurs la stabilité et la résistance de la banque à des chocs économiques. 


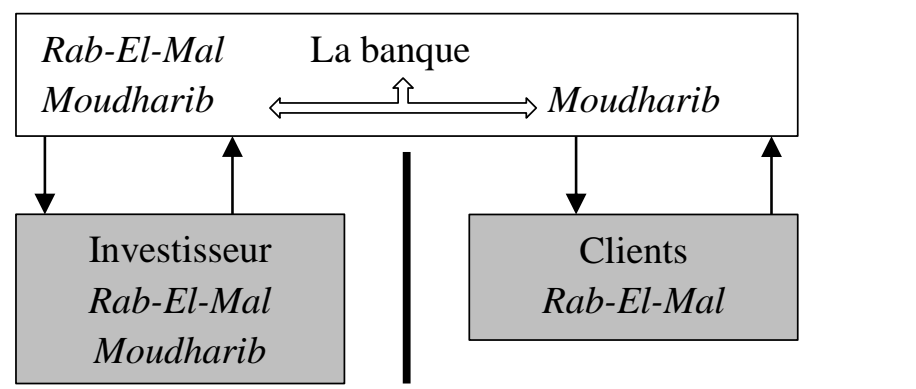

Figure 1 : modèle pur de banque islamique

\section{Modèle hybride ou commercial (finance non-participative)}

Dans ce cas, une opération de partage des profits et des pertes (PPP) est toujours appliquée au passif du bilan avec le Moudharaba. En revanche, elle se combine à l'Actif avec des opérations commerciales non-PPP et adossées à des actifs tangibles, à savoir notamment le Mourabaha, l'Istisna, le Salam et l'Ijara (Bitar et Madiès, 2013).

Ce modèle plus flexible en matière d'instruments proposés est considéré comme moins conforme à la Charia puisqu'il favorise les opérations non-PPP (Mark-up financing) par rapport aux opérations PPP. Dans ce modèle, l'Actif est plus diversifié avec une palette de contrats avec différentes échéances et niveaux de risque.

En premier lieu, ce modèle offre le contrat du Mourabaha comme un instrument d'investissement à court terme (El-Hawary, Grais et Iqbal, 2007). Il s'agit d'une alternative islamique au crédit bancaire. Le contrat du Mourabaha fait intervenir trois acteurs : (i) le cliententrepreneur qui souhaite acheter un bien, (ii) la banque islamique qui achète le bien sur demande et selon les instructions de son client-entrepreneur, et (iii) le vendeur du bien. La banque islamique revend le bien à son client avec une marge de profit avec paiement immédiat ou échelonné. Toutefois, l'actif acheté demeure propriété de la banque jusqu'au règlement du dernier versement. En second lieu, une banque islamique peut proposer des instruments à moyen terme comme l'Ijara et l'Istisna. Pour le long terme, les banques islamiques peuvent 
recourir au Moudharaba ou au Mousharaka, toutefois rarement utilisés dans un modèle hybride.

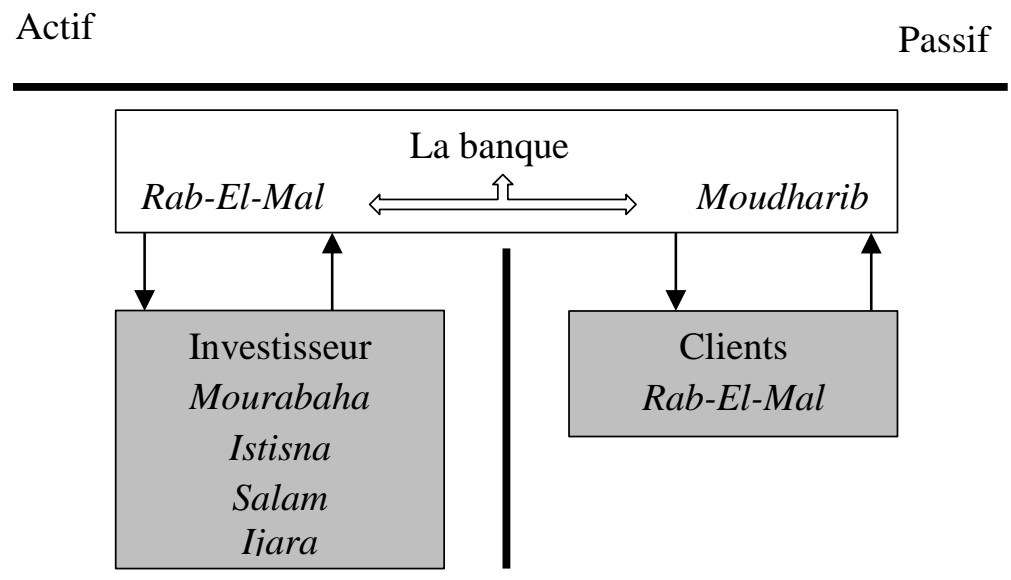

Figure 2 : modèle hybride de banque islamique

Ces deux modèles d'intermédiation illustrent bien le débat sur l'identité des banques islamiques. Selon les savants de la finance islamique, le modèle pur ou participatif est à privilégier par rapport au modèle hybride non-participatif. Cependant, ce dernier modèle peut être accepté pour une période donnée et transitoire ou dans certaines situations où son application est jugée adéquate (Sundararajan et Errico, 2002 ; Khan, 2010). En outre, des auteurs, comme Siddiqui (2002), expliquent qu'il s'agit d'une question de degré et que des opérations non-PPP peuvent être considérées comme conforme à la Charia s'il y a un minimum de partage de profit et de perte entre les deux parties du contrat. Enfin, El Gamal (2002) explique qu'il n'y a pas de justification coranique pour préférer un mode de financement à l'autre. Ainsi, cette préférence n'est qu'une inclination personnelle de l'observateur.

\section{Quel type d'intermédiation les banques islamiques privilégient-elles ?}

La réponse à cette question ne peut être qu'empirique et l'idée est de savoir quel modèle, parmi les deux modèles théoriques mentionnés au-dessus, est le plus cohérent avec la pratique actuelle d'intermédiation des banques islamiques. Le tableau 1 fournit des informations sur la 
part des différentes activités, jugées conformes à la Charia, des banques islamiques à l'actif de leur bilan.

\section{Tableau 1}

Le système bancaire Islamique : catégories d'activités des banques islamiques à l'Actif (en \% du total des activités conformes à la Charia)

\begin{tabular}{lcccc}
\hline & $2000-2007$ & $2008-2009$ & $2010-2011$ & $2000-2011$ \\
\hline Opérations PPP & 2.34 & $\mathbf{3 . 0 5}$ & 2.54 & 2.49 \\
Moudharaba & $\mathbf{2 . 3 5}$ & $\mathbf{3 . 3 6}$ & $\mathbf{4 . 6 2}$ & $\mathbf{2 . 9 0}$ \\
Mousharaka & 4.69 & 6.41 & 7.12 & 5.39 \\
\hline Total opérations PPP & & & & \\
\hline Opérations adossées à des actifs & $\mathbf{8 1 . 0 9}$ & $\mathbf{7 9 . 5 8}$ & $\mathbf{7 5 . 1 2}$ & $\mathbf{7 9 . 8 5}$ \\
Mourabaha & 4.60 & 11.69 & 10.73 & 6.8 \\
Ijara & 2.22 & 1.98 & 1.32 & 2.03 \\
Istisna & 1.27 & 0.03 & 0.22 & 0.89 \\
Salam & 89.18 & 93.28 & 87.39 & 89.57 \\
\hline Total opérations commerciales & & & & \\
\hline Opérations rémunérées par des frais & & 0.13 & 0.87 & 0.8 \\
Qard Hasan & 0.96 & 0.18 & 4.62 & 4.24 \\
\hline Autres opérations & 5.17 & l'Islamic Banks and Financial Institutions Information (IBIS).
\end{tabular}

Le tableau 1 témoigne bien du rôle prépondérant des opérations commerciales (i.e. opérations adossées à des actifs) qui représentent en moyenne, sur la période 2000-2011, 90 \% des activités des banques islamiques. Les contrats de Moudharaba et Mousharaka ne constituent sur la même période que $5 \%$ respectivement des activités des banques islamiques. On notera que le contrat de Mourabaha, qui représente $80 \%$ des activités des banques islamiques, prédomine au sein des opérations commerciales. Ainsi, les contrats d'Ijara, d'Istisna et de Salam sont des opérations adossées à des actifs beaucoup plus marginales même si les opérations d'Ijara représentent à elles seules un peu plus que l'ensemble des opérations PPP.

D'ailleurs, Khan (2010) montre qu'en 2006 les plus grandes banques islamiques (e.g. banque Al-Rajhi, Kuwait Finance House, banque Islamique de Dubaï, banque Al-Baraka, etc.) 
se basent principalement sur des opérations commerciales. En utilisant la base de données IBIS ainsi que les données financières du site web de The Asian Banker, nous exposons dans l'annexe 2 le classement des plus grandes banques islamiques et la répartition de leurs opérations entre opérations commerciales (i.e. non-profit loss sharing arrangements, non-PLS) et opérations de partage de profits et de pertes (i.e. profit loss sharing arrangement, PLS). Ce tableau montre clairement la domination des opérations de Mourabaha sur les trois périodes d'avant, pendant et d'après crise financière.

Ces chiffres conduisent à nous interroger sur la nature de l'intermédiation des banques islamiques. Une banque, qui dépend excessivement des activités non-PPP et s'en sert comme principal véhicule de développement, risque d'être considérée comme une banque "non pratiquante » s'éloignant des préceptes de la finance islamique (Khan, 2010; Abedifar, Molyneux et Tarazi, 2013; Beck, Demirgüç-Kunt and Merrouche, 2013). Ainsi, Khan (2010) relate une décision de la Cour fédérale pakistanaise de la Charia contre l'existence des banques islamiques qui privilégient le Mourabaha, contrat jugé comme comportant un intérêt déguisé. Cette cour a exigé une conversion complète du système bancaire à la finance participative. Rappelons que celle-ci privilégie les opérations fondées sur le partage des profits et des pertes, la prohibition de l'intérêt et surtout la diffusion des valeurs humaines et éthiques au lieu de la maximisation des gains et des profits.

Pour une évaluation plus générale, nous présentons dans le tableau 2 la part des opérations de type islamique (i.e. opérations commerciales et opérations PPP déjà présentées dans le tableau 1) dans le bilan total des banques islamiques. Les instruments islamiques constituent en moyenne $50 \%$ du total des activités de ces banques sur la période 2000-2011, $80 \%$ de ces activités islamiques passant par le contrat Mourabaha. En outre, les disponibilités et les activités d'investissements (en actifs financiers et immobiliers) représentent respectivement $27 \%$ et $19 \%$ du total de l'Actif. D'ailleurs, ces résultats montrent que les banques islamiques possèdent un montant élevé d'actifs liquides. Cela s'explique par le fait que ces institutions ne jouissent pas des mêmes facilités à se refinancer et donc à trouver des liquidités que les banques conventionnelles. En particulier, comme elles ne peuvent se refinancer sur le marché interbancaire classique, elles s'appuient sur les comptes d'investissement à long terme à partir desquels elles financent leur développement (Metwali, 1997). En outre, elles ne peuvent ni céder leurs dettes (Beck, Demirgüç-Kunt, et Merrouche, 2013), ni avoir des relations avec les banques conventionnelles (Akhtar, Ali et Sadaqat, 2011), 
ou bien encore recourir à la Banque centrale comme prêteur en dernier ressort à cause des contraintes imposées par la Charia.

\section{Tableau 2}

Part relative des différentes composantes de l'actif des banques islamiques comprenant les opérations islamiques et non-islamiques (en \% du total des actifs).

$$
2000-2007 \quad 2008-2009 \quad 2010-2011 \quad 2000-2011
$$

\begin{tabular}{lcccc} 
Disponibilités (actifs liquides) & 28.22 & 25.65 & 22.46 & 26.71 \\
\hline Opérations de banque islamique & $\mathbf{4 8 . 5 9}$ & $\mathbf{4 8 . 6 6}$ & $\mathbf{5 0 . 8 5}$ & $\mathbf{4 9 . 0 1}$ \\
\hline Portefeuille d'investissements & 17.18 & 19.75 & 22.34 & 18.59 \\
\hline & & & & \\
\hline Autres & 6.01 & 5.94 & 4.35 & 5.69 \\
\hline
\end{tabular}

Source : calculs des auteurs à partir des données de l'Islamic Banks and Financial Institutions Information (IBIS).

Enfin, le tableau 3 s'intéresse à la nature des ressources utilisées par les banques islamiques et leur conformité à la Charia. Conformément aux trois modèles des banques islamiques exposés ci-dessus, nous constatons que les comptes d'investissements affectés et standards représentent en moyenne un peu plus de $50 \%$ des ressources des banques islamiques sur la période 2000-2011 et constituent donc leur principale ressource. Ces comptes se basent sur le contrat de type Moudharaba entre les clients-déposants et la banque où il existe un partage des pertes et des profits entre les deux partenaires. Tout de même, on se doit de constater que les comptes courants (nommés Wadiah ou Amanah) et les comptes d'épargne constituent près du tiers des dépôts sur la période 2000-2011. Or ces comptes sont très proches de ce que proposent les banques classiques. 


\section{Tableau 3}

Le système bancaire Islamique : les différentes catégories de dépôts (en \% du total des dépôts)

$2000-2007 \quad 2008-2009 \quad 2010-2011 \quad 2000-2011$

Comptes courants et d'épargne

32.74

27.80

28.04

30.99

Les comptes d'investissements

52.34

53.55

53.56

52.79

Autres

14.92

18.65

18.40

16.22

Source : Islamic Banks and Financial Institutions Information (IBIS) ; calculs des auteurs.

\section{Comment l'intermédiation des banques islamiques influe-t-elle sur leurs fonds propres réglementaires? Une étude économétrique comparative entre banques islamiques et banque conventionnelles}

Compte tenu de la nature des activités des banques islamiques, nous nous intéressons maintenant à la relation entre les comptes d'investissements (i.e. structure du passif du bilan) ${ }^{\mathrm{iii}}$, les opérations commerciales (i.e. structure de l'actif du bilan) ${ }^{i v}$ et la capitalisation des banques islamiques comparativement aux banques conventionnelles. En nous appuyant sur les bases de données IBIS et Bankscope, nous proposons d'étudier ici ces relations grâce à des statistiques descriptives puis des modèles économétriques.

\section{Fonds propres réglementaires : quelques statistiques descriptives}

Comme en atteste le graphique 1, entre 2005 et 2011, les différents indicateurs de capitalisation $^{\mathrm{v}}$, à savoir les ratios de fonds propres de base (T1RP et T1TAP) et les ratios intégrant au numérateur l'ensemble des fonds propres (TCRP et TCTAP) sont sur un trend stable ou légèrement décroissant ${ }^{\mathrm{vi}}$. La stabilité du niveau relatif des fonds propres réglementaires provient du fait que les banques islamiques présentent sur la période d'étude (2005-2011) une répartition stable entre opérations PPP (Moudharaba) et opérations adossées à des actifs (Mourabaha). En outre, nous trouvons que l'utilisation des comptes d'investissements n'a pas poussé les banques islamiques à prendre plus de risque (en 
investissant massivement dans des activités liées aux opérations de PPP -modèle pur, voir supra-). Au contraire, ces banques continuent de profiter davantage des instruments adossés à des actifs (cf. tableau 1 et graphique 1) dont le risque est moins fortement pondéré dans le calcul des ratios de capital réglementaires.

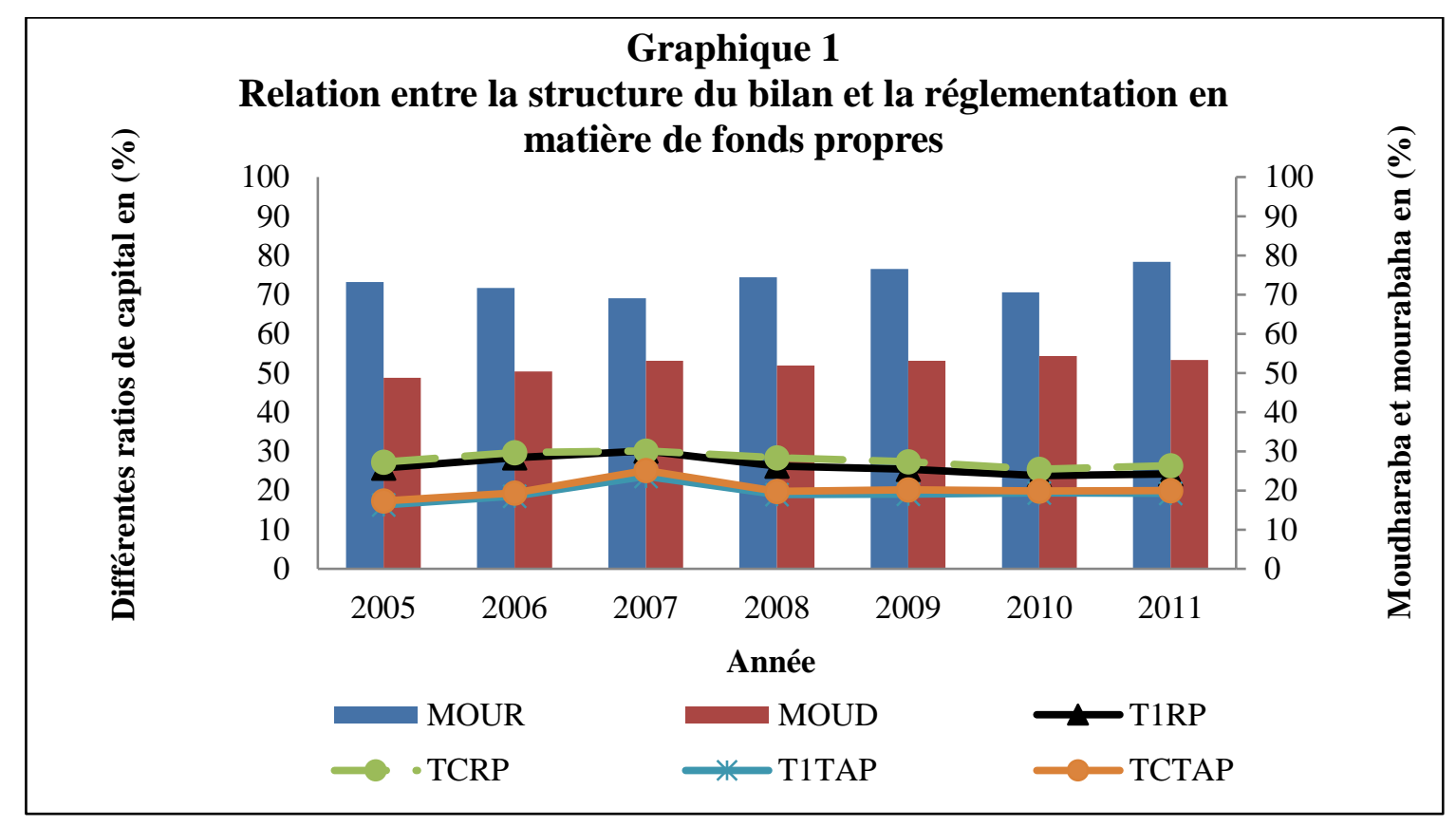

Source : Bankscope et calculs des auteurs.

Le graphique 2, ci-dessous, montre que les banques islamiques sont plus capitalisées que les banques conventionnelles. Plusieurs raisons peuvent expliquer cette meilleure capitalisation et pourquoi une réglementation du capital est absolument nécessaire pour les banques islamiques aussi. Selon l'Institut Islamique de Recherche et de Formation (IIRF), ces raisons sont au nombre de trois : (i) la volonté de renforcer la stabilité du système bancaire grâce à une meilleure capitalisation, (ii) compenser le faible accès des banques islamiques au marché interbancaire et l'incapacité à profiter de fonds propres complémentaires à cause des contraintes imposées par la Charia concernant le paiement d'intérêt et (iii) protéger les détenteurs de dépôts à vue d'une faillite bancaire. D'ailleurs, nous confirmons économétriquement, dans la partie suivante, que les banques islamiques sont plus capitalisées que les banques conventionnelles et nous en expliquons plus en détails les raisons. 


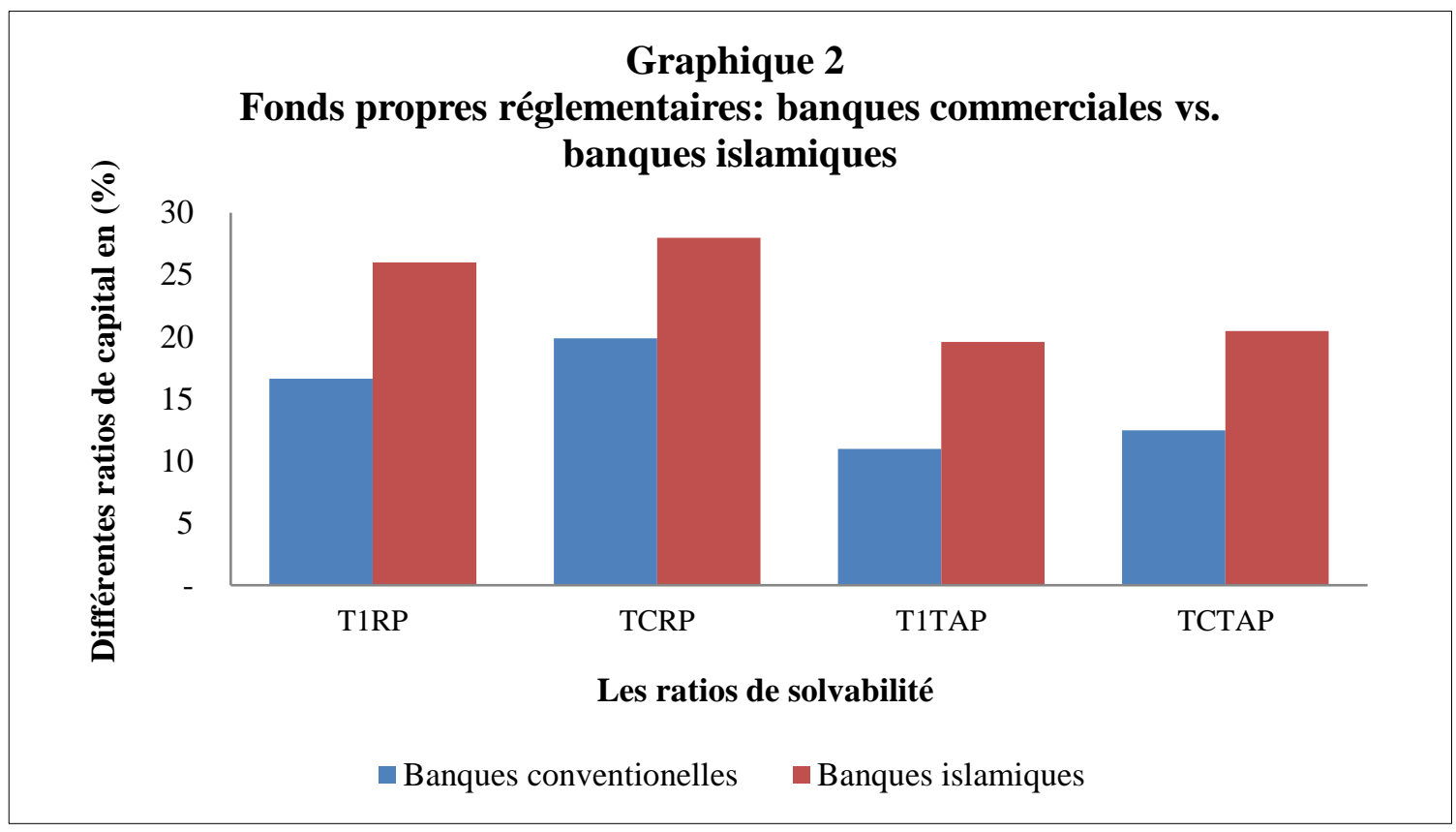

Source : IBIS, Bankscope et calculs des auteurs.

\section{Fonds propres réglementaires : banques islamiques versus banques conventionnelles}

Afin de consolider les résultats de la partie précédente, nous effectuons ici une régression linéaire multiple en contrôlant les effets fixes des années et des pays. Notre échantillon est composé de 656 banques (dont 116 banques sont islamiques) issues de la base de données Bankscope sur la période 1999 - 2013. Nous avons choisi de travailler avec des banques de 33 pays où les deux types de banques coexistent (Abedifar, Molyneux et Tarazi, 2013 ; Beck, Demirgüç-Kunt, et Merrouche, 2013).

Nous utilisons le modèle de régression suivant :

$$
\mathrm{CAP}_{\mathrm{ijt}}=\alpha+\beta_{1} \times \mathrm{IBDV}+\beta_{2} \times \mathrm{CONTROLE}_{\mathrm{ijt}}+\varepsilon_{\mathrm{ijt}}
$$

Le tableau 4 présente nos résultats économétriques. Les p-values sont calculées a) en corrigeant le problème d'hétéroscédasticité (afin de réduire la sensibilité de la régression à des valeurs extrêmes), et b) en les ajustant de la corrélation des erreurs intragroupes définie au niveau de la banque (Cluster at the bank level, selon Anginer et Demirgüç-Kunt, 2014). La construction de notre modèle de régression nous permet d'éviter le problème de multicolinéarité parce que nous avons régressé une variable binaire-IBDV- (égale 1 si la banque est islamique, 0 sinon) sur l'ensemble des variables d'intérêt pour notre étude. ${ }^{\text {vii }}$ 
Notre tableau de régression utilise différents ratios permettant d'appréhender les variables étudiées. Le modèle étudiant la capitalisation respective des banques islamiques et conventionnelles montre une relation positive et significative pour l'ensemble des ratios mesurant cette variable de capital. ${ }^{\text {viii }}$ Ceci atteste du fait que les banques islamiques sont plus capitalisées que les banques conventionnelles. Dans un environnement concurrentiel, les banques islamiques sont incitées à redistribuer des profits suffisants afin d'éviter la fuite de leurs clients vers les réseaux bancaires traditionnels. Cependant, une telle politique encourage les dirigeants des banques à prendre plus de risques - en profitant des comptes d'investissements comme levier financier - puisque le taux de profit annoncé aux détenteurs des comptes d'investissement ne reflète pas ce que la banque est en mesure d'offrir. Cela oblige les banques islamiques à renforcer leur capitalisation, ce qu'elles n'auraient normalement pas à faire si elles appliquaient à la lettre le principe du PPP (modèle pur) et faisaient assumer les risques uniquement par leurs clients-investisseurs.

Ensuite, le modèle des fonds propres complémentaires ou quasi-capital montre que les banques islamiques possèdent moins des fonds propres complémentaires que les banques conventionnelles $^{\mathrm{ix}}$. Les fonds propres complémentaires incluent certaines réserves, des instruments hybrides et des titres de dette subordonnée. Harzi (2012) explique ce résultat par le fait que les banques ne peuvent verser d'intérêts et donc se privent d'un certain nombre d'instruments financiers qui s'intègrent au capital complémentaire.

Enfin, notre modèle inclut cinq variables de contrôle qui nous permettent d'avoir des résultats complémentaires.

La variable taille (logarithme du total des actifs) montre que les grandes banques sont moins capitalisées quel que soit le ratio retenu. En effet, des grandes banques préfèrent avoir un ratio de capital moins élevé et un levier financier plus fort parce qu'elles possèdent un niveau important de profit retenus. Ce profit peut jouer le rôle d'une réserve supplémentaire de capital. Ainsi, les grandes banques préfèrent investir leurs fonds dans des projets profitables au lieu de détenir un ratio de capital élevé qui traduit une inefficience dans l'activité de transformation d'échéances des banques et la détention de ressources «dormantes ». Finalement, les grandes banques sont plus efficientes et moins risquées parce qu'elles profitent d'effet d'expérience (économie de l'échelle et effet d'apprentissage). En outre, elles possèdent des informations plus nombreuses et plus fines sur l'état financier de leurs clients étant donné le degré de sophistication plus élevé de leurs systèmes d'information. Elles ont aussi une plus forte 
expertise en matière de gestion des risques et du risque de crédit que des petites banques. Pour ces raisons, les autorités réglementaires sont plus flexibles en matière de fonds propres avec les grandes banques qu'avec les plus petites.

La variable crédit/total actifs montre une relation positive et significative avec la partie quasi-capital du ratio du capital et négative et significative avec le ratio global de capital. Cela signifie que les banques les plus capitalisées octroient moins de crédits que les banques les moins capitalisées. Aussi, ces résultats signifient que l'engagement dans des activités bancaires traditionnelles requiert moins de capital réglementaire.

En ce qui concerne le ratio autres revenus/total actif, nous avons trouvé une relation positive et significative avec les différents ratios de capitalisation à l'exception des ratios des fonds propres complémentaires. Nous observons que l'engagement dans des activités d'investissement immobilier impose aux banques un niveau de capitalisation plus élevé. Cela peut être expliqué par le fait que l'orientation vers des activités d'investissements non traditionnelles rend les banques plus risquées. Ce risque doit être compensé par un niveau de capital plus élevé.

Enfin, le ratio de marge d'intérêt nette est positivement associé aux ratios de capital ce qui signifie que les banques les plus capitalisés sont les plus profitables.

Pour vérifier la robustesse de nos résultats, nous avons décomposé notre échantillon entre grandes banques, banques de taille moyenne et petites banques. Nous avons aussi comparé les banques selon leur présence régionale. Enfin, nous avons étudié le degré de capitalisation des banques islamiques pour la période de crise financière (2007-2009), d'avant-crise et d'après la crise. Les résultats sont exposés dans les tableaux 5, 6 et 7, respectivement.

Dans le tableau 5, les modèles de fonds propres de base et d'adéquation du capital montrent que les banques islamiques de grande taille et de taille moyenne ne différent pas des banques conventionnelles en terme de capitalisation. Aussi, le modèle de fonds propres complémentaires montre que les banques islamiques de taille moyenne possèdent moins de quasi-capital que les banques conventionnelles.

Néanmoins, les petites banques islamiques révèlent des différences significatives avec les banques conventionnelles de petite taille. D'abord, les modèles de fonds propres de base montrent que les petites banques islamiques sont plus capitalisées que les banques 
conventionnelles. ${ }^{\mathrm{x}}$ En outre, nous observons les mêmes résultats avec le modèle d'adéquation du capital. De surcroit, les résultats du modèle de fonds propres complémentaires attestent bien que les petites et moyennes banques islamiques utilisent moins d'instruments hybrides et de titres de dette subordonnée que les banques conventionnelles parce que ces instruments sont difficilement conformes avec la Charia.

Ces résultats traduisent un écart significatif entre les grandes banques islamiques, les banques islamiques de taille moyenne et les petites banques islamiques (Beck, Demirgüç-Kunt, et Merrouche, 2013). Notamment, nous avons pu trouver que les petites banques islamiques étaient plus capitalisées que les grandes banques islamiques. Plus précisément, les résultats montrent clairement que les petites banques islamiques jouent un rôle prépondérant dans cette différence entre les deux types de banques. Ensuite, la section 1 a pu mettre en avant que les banques islamiques préféraient un modèle d'intermédiation privilégiant les opérations non-PPP pour offrir des produits et des services proches de ceux proposées par les banques conventionnelles - les banques islamiques profitent des comptes d'investissements au passif du bilan pour investir dans des activités de Mourabaha à l'actif de leur bilan -.

Cela signifie que le modèle d'intermédiation des petites banques islamiques (i.e. modèle non pur) les rend plus capitalisées que le reste des banques islamiques et que les banques conventionnelles. Cette différence peut être expliquée par le fait que les petites banques islamiques sont plus prudentes en matière d'activités non-PPP. Elles sont aussi plus jeunes, moins expérimentées et possèdent moins d'instruments de gestion de risque en comparaison avec les grandes banques islamiques et leurs homologues conventionnelles. Cela explique qu'elles aient des matelas de sécurité en matière de fonds propres plus importants. D'ailleurs, les ratios de fonds propres complémentaires attestent bien que les petites banques islamiques et aussi les banques islamiques de taille moyenne - sont beaucoup moins outillées (en matière d'instruments hybrides et de dette subordonnée) que les banques conventionnelles.

Au-delà de la variable taille, nous avons mené une régression en tenant compte des différentes régions auxquelles appartiennent les banques de notre échantillon ${ }^{\mathrm{xi}}$. Pour ce faire, nous avons régressé notre variable binaire (IBDV) sur l'ensemble de nos variables explicatives en faisant le produit avec les cinq variables d'intérêt. ${ }^{x i i}$ Les résultats sont présentés dans le tableau 6 et témoignent d'une certaine diversité régionale. Nous trouvons que les banques islamiques des pays du GCC jouissent d'un niveau élevé de capital. Etant donné la richesse des pays du golf liée à leur rente pétrolière, les banques n'éprouvent pas de difficultés à lever du 
capital et à renforcer donc leurs capitaux propres. Aussi, une mauvaise gestion du risque de ces banques combinée à des contraintes imposées par la Charia sur le levier financier (investissements dans des produits dérivés, la vente de dettes, le principe des opérations adossées à des actifs, etc.) rendent difficile l'investissement de ressources collectées dans des projets profitables et pourrait se traduire par une bonne capitalisation.

Concernant les autres régions, nous trouvons que les banques dans les pays des zones MENA, SEA et EU ne sont pas différentes de leurs homologues conventionnelles sauf en matière de fonds propres complémentaires. Nous avons trouvé que les banques islamiques utilisent moins de fonds propres complémentaires dans les zones GCC et l'EU en comparaison avec les banques conventionnelles. Toutefois, les banques islamiques d'Afrique subsaharienne sont moins capitalisées - en termes de fonds propres de base, fonds propres complémentaire et d'adéquation du capital - que les banques conventionnelles.

Pour conclure, les différences entre banques islamiques et banques conventionnelles dépendent de la région concernée ainsi que de la taille des banques. Cependant, il est important de noter que les différences les plus marquées apparaissent dans la région GCC alors que les autres régions en sont exemptes. Ainsi, les banques islamiques, en se rapprochant du modèle d'intermédiation des banques conventionnelles, sont très proches les unes des autres en matière de capital et cela dans les régions MENA, SEA et EU.

Enfin, nous avons régressé nos résultats sur la période d'avant crise (1999-2006), de crise (2007-2009) et d'après la crise (2010-2013). Les résultats, présentés dans tableau 7, montrent clairement que les banques islamiques sont plus capitalisées que les banques conventionnelles, et cela particulièrement pendant la période de crise et d'après la crise. En outre, nous montrons que les banques islamiques possèdent moins de fonds propres complémentaires sur les trois périodes. Cela peut expliquer la meilleure résistance des banques islamiques pendant la crise parce qu'elles sont non seulement plus capitalisées que les banques conventionnelles mais aussi mieux capitalisées en possédant moins de fonds propres complémentaires de moins bonne qualité que les fonds propres de base. 


\section{Conclusion}

Au regard de la grille de lecture que constituent les deux modèles d'intermédiation utilisables par une banque pour être islamique, il apparaît que les banques islamiques, telles que observées de manière agrégée sur la période 2000-2011, combinent des opérations typiquement islamiques avec des opérations classiques. A l'actif, les opérations intrinsèquement islamiques sont marginales alors qu'elles sont majoritaires côté passif même si les dépôts classiques sur compte courant et d'épargne représentent près du tiers des ressources des banques islamiques. Le caractère islamique des banques islamiques peut donc être clairement questionné.

Pour autant, à partir d'un échantillon de 656 banques conventionnelles et banques islamiques, nous avons pu démontrer que les banques islamiques étaient plus capitalisées que les banques conventionnelles. En outre, en matière de fonds propres, le modèle hybride d'intermédiation des banques islamiques présente un écart significatif avec celui des banques conventionnelles lorsque les petites banques islamiques et les banques de la région GCC sont regardées de plus près. En revanche, nulle différence significative n'est observée lorsqu'on examine les grandes banques islamiques ou les banques des régions autres que la région GCC. Enfin, nous avons montré que les banques islamiques avaient été plus capitalisées pendant la crise financière de 2007-2009, ce qui peut expliquer leur meilleure résistance.

Les banques islamiques, en s'orientant vers des activités où elles cherchent à maximiser leurs profits plutôt qu'à partager leurs profits et leurs pertes (PPP) et où elles proposent de manière détournée un taux d'intérêt comme les banques conventionnelles, sont devenues vulnérables, moins profitables, et risquent de ne pas rémunérer les détenteurs des comptes d'investissement à un même taux que celui proposé par les banques conventionnelles. Cela peut engendrer des effets déstabilisants. Pour se prémunir, elles lissent le taux de rendement proposé sur les comptes d'investissement en utilisant des fonds de réserves (IRR et PER). De surcroît, les banques islamiques, malgré leur spécificité au passif, ont besoin d'appliquer les réglementations de Bâle III en matière de capital réglementaire parce que leur modèle d'intermédiation plutôt basé sur les opérations adossées à des biens tangibles est largement « benchmarqué » sur celui des banques conventionnelles. 
Annexe 1

Les principaux organismes réglementaires des banques islamiques et les différentes catégories d'institutions Financières Islamiques

Type Objectifs et type d'activité

d'institution

Organismes réglementaires internationaux

IFSB Islamic Financial Services Board

Promouvoir la transparence, la coopération et la coordination dans le but de standardiser la réglementation entre, d'une part, les différentes institutions financières islamiques et d'autre part le reste du système financier.

AAOIFI Auditing and Accounting Organisation of Islamic Financial Institutions

Préparation et adaptation des différents standards de comptabilité, d'audit, de gouvernance, d'éthique et de Charia aux institutions financières islamiques. L'AAOIFI est considérée avec l'IFSB comme le comité de Bâle des banques islamiques.

$I D B$ Islamic Development Bank

Amélioration et promotion du développement économique et sociales des pays musulmans. Elle est considérée comme la banque mondiale des pays islamiques

CIBAFI General Council for Islamic Banks and Financial Institutions

Définition et développement des produits financiers islamiques conformes avec la Charia. CIBAFI s'intéresse aussi au développement des ressources humaines ainsi qu'à l'information et à l'analyse financière liée à cette industrie.

LMC Liquidity Management Center

La création et le développement d'un marché monétaire interbancaire pour investir les surplus de liquidité à court et moyen terme des banques islamiques. Il s'agit de mettre en place des opérations conformes à la Charia sur ce marché

IIRA Islamic International Rating Agency

Reconnue par la banque islamique de développement, son objectif principal comme agence de notation est d'évaluer les risques liés aux produits financiers islamiques pour les rendre transparents sur les marchés de capitaux.

AIBIM Association of Islamic Banking Institutions in Malaysia

Son objectif est la mise en place de pratiques saines qui vise à promouvoir le système bancaire islamique en améliorant le niveau d'expertise des cadres et des employés des banques islamiques en Malaisie.

Institutions financières islamiques

Banques C'est sont des institutions financières conformes à la Charia. Leurs activités de financement se basent sur des opérations

Islamiques $\quad$ commerciales et des opérations de partage de profits et de pertes (PPP). Elles sont au nombre de 219 selon l'AIBIM.

Agences Il s'agit des agences Islamiques ouvertes au sein des grandes banques commerciales conventionnelles. Ces dernières

islamiques les considèrent comme une opportunité pour attirer les fonds de clients musulmans. L'AIBIM évalue à 170 le nombre d'institutions financières conventionnelles avec un guichet islamique.

Compagnies Selon l'IFSB, l'assurance conforme à la Charia ou "Takaful » est "l'équivalent islamique de l'assurance classique,

d'assurance offrant à la fois des produits d'assurance-vie (ou «famille ») et d'assurance dommages ». Selon AIBIM, il y a 132 entreprises d'assurance islamique Takaful.

Sociétés de $\quad$ Aider les gens ainsi que les très petites entreprises dans une logique d'économie sociale et solidaire. Par exemple, la micro-finance $\quad$ société Qard El Hassan au Liban.

Source : Divers ; les auteurs. 
Annexe 2

Répartition des opérations des plus grandes banques islamiques entre PPP et non-PPP pour la période 2000 - 2011.

\begin{tabular}{|c|c|c|c|c|c|c|c|c|c|c|}
\hline & \multirow{3}{*}{$\begin{array}{l}\text { Classement } \\
\text { The } \\
\text { Banker } 2011 \\
\end{array}$} & \multicolumn{3}{|c|}{$2000-2007$} & \multicolumn{3}{|c|}{$2008-2009$} & \multicolumn{3}{|c|}{$2010-2011$} \\
\hline & & \multicolumn{2}{|c|}{$\%$ PPP } & \multirow{2}{*}{$\begin{array}{c}\% \text { Non-PPP } \\
\text { Mourabaha }\end{array}$} & \multicolumn{2}{|c|}{$\%$ PPP } & \multirow{2}{*}{$\begin{array}{l}\text { \% Non-PPP } \\
\text { Mourabaha }\end{array}$} & \multicolumn{2}{|c|}{$\%$ PPP } & \multirow{2}{*}{$\begin{array}{c}\text { \% Non-PPP } \\
\text { Mourabaha }\end{array}$} \\
\hline & & Moudharaba & Mousharaka & & Moudharaba & Mousharaka & & Moudharaba & Mousharaka & \\
\hline & & \multicolumn{9}{|c|}{ Moyen-Orient et Afrique du Nord (MENA2) } \\
\hline Asie & & & & & & & & & & \\
\hline Bank Asya 21 & 21 & -- & -- & 82.67 & -- & -- & 82.36 & -- & -- & 96.62 \\
\hline Jordan Islamic bank 44 & 44 & 0.01 & 3.53 & 98.18 & -- & 1.48 & 87.15 & -- & 1.33 & 85.90 \\
\hline Meezan bank 60 & 60 & -- & 7.77 & 62.59 & -- & 15.07 & 61.76 & -- & 28.56 & 42.23 \\
\hline Tadhamon International 61 & 61 & 13.09 & 1.69 & 83.62 & 0.47 & 24.54 & 66.88 & 24.63 & 13.26 & 58.87 \\
\hline Arab Islamic bank 92 & 92 & 16.68 & -- & 69.73 & 6.55 & -- & 45.88 & 4.76 & -- & 49.94 \\
\hline \multicolumn{11}{|l|}{ Afrique } \\
\hline Faisal Islamic bank 31 & 31 & -- & -- & 99.90 & -- & -- & 99.90 & -- & -- & 86.37 \\
\hline Bank of Khartoum 63 & 63 & 7.16 & 18.04 & 65.58 & 3.43 & 7.84 & 71.67 & 4.89 & 5.94 & 60.97 \\
\hline Faisal Islamic Sudan 69 & 69 & -- & 14.03 & 72.23 & 0.17 & 2.61 & 69.51 & 10.71 & 3.24 & 56.56 \\
\hline Al-Baraka Tunisia 88 & 88 & -- & -- & 72.12 & -- & -- & 97.24 & -- & -- & 99.90 \\
\hline & & \multicolumn{9}{|c|}{ Conseil de coopération des pays du Golfe (GCC) } \\
\hline Al-Rajhi Bank 3 & 3 & -- & -- & 89.59 & -- & -- & 99.90 & -- & -- & 99.90 \\
\hline Kuwait Finance House 5 & 5 & -- & -- & 87.99 & -- & -- & 77.02 & -- & -- & 85.54 \\
\hline Dubai Islamic bank 8 & 8 & 5.49 & 8.29 & 64.22 & 19.54 & 11.97 & 35.28 & 6.73 & 14.43 & 26.00 \\
\hline Abu Dhabi Islamic bank 9 & 9 & 4.14 & 0.25 & 68.35 & 6.75 & 0.12 & 51.48 & 4.68 & -- & 47.02 \\
\hline Al-Baraka Bahrain 13 & 13 & 3.87 & 1.50 & 91.94 & 2.77 & 4.06 & 90.06 & 3.43 & 8.72 & 82.35 \\
\hline \multirow[t]{2}{*}{ Qatar Islamic bank 14} & 14 & 3.55 & 0.26 & 78.71 & 5.84 & 0.13 & 59.89 & 3.13 & 0.20 & 67.08 \\
\hline & & \multicolumn{9}{|c|}{ Iran } \\
\hline Bank Melli 1 & 1 & -- & -- & 94.56 & -- & -- & 100 & -- & -- & 100 \\
\hline Banl Mellat 2 & 2 & -- & -- & 99.90 & -- & -- & 99.90 & -- & -- & 99.90 \\
\hline Bank Saderat 4 & 4 & -- & -- & 92.39 & 6.97 & -- & 68.39 & 4.57 & -- & 81.70 \\
\hline \multirow[t]{2}{*}{ Bank Sepah 7} & 7 & -- & -- & 100 & -- & -- & 100 & -- & -- & 100 \\
\hline & & \multicolumn{9}{|c|}{ Sud-Est de l'Asie (SEA) } \\
\hline Bank Rakyat Malaysia 10 & 10 & -- & -- & 94.82 & -- & 0.04 & 97.10 & -- & 0.14 & 98 \\
\hline \multirow{2}{*}{ Bank Syariat Madiri 64} & 64 & 7.27 & 13.42 & 79.18 & 22.82 & 21.15 & 53.83 & 15.79 & 17.62 & 55.38 \\
\hline & & \multicolumn{9}{|c|}{ Union Européenne (UE) } \\
\hline $\begin{array}{l}\text { Bank of London and the Middle } \\
\text { East } 61\end{array}$ & 61 & 0.73 & 0.90 & 43.82 & 2.77 & 8.26 & 90.06 & 3.43 & 8.72 & 82.35 \\
\hline
\end{tabular}




\section{Bibliographie}

[1] Abedifar, P., Molyneux P., et Tarazi, A., (2013), «Risk In Islamic Banking », Review of Finance 17, 2035-2096.

[2] Akhtar, M. F., Ali, K., et Sadaqat, S., (2011), «Factors influencing the profitability of Islamic banks of Pakistan », International Research Journal of Finance and Economics $66,125-132$.

[3] Anginer, D. et Demirgüç-Kunt, A., (2014), « Bank capital and systemic stability », Policy Research Working Paper No. 6948, The World Bank, Washington, DC.

[4] Beck, T., Demirgüç-Kunt, A., et Merrouche, O., (2013), « Islamic vs. Conventional Banking: Business Model, Efficiency and Stability », Journal of Banking \& Finance, $37,433-447$.

[5] Bitar, M., et Madiès, P., (2013), «Les spécificités des banques islamiques et la réglementation de BÂLE III », revue d'économie financière 111, 293-310.

[6] El Gamal, M.A., (2000), « A Basic Guide to Contemporary Islamic Banking and Finance », Islamic Society of North America, (accédé le 06.7.2015) http://www.nubank.com/islamic/primer.pdf.

[7] El-Hawary D., Grais W., et Iqbal Z., (2007), « Diversity in the Regulation of Islamic Financial Institutions », The Quarterly Review of Economic and Finance, 46, 778-800.

[8] Errico et Farahbaksh, (1998), «Islamic Banking: Issues in Prudential Regulations and Supervision », International Monetary Fund Working Paper, WP/98/30

[9] Ernest \& Young, (2012), The World Islamic Banking Competiveness Report 2013.

[10] Hassan, M., et Dridi, J., (2010), « The effects of the Global Crisis on Islamic and conventional banks: A comparative study », International Monetary Fund Working Paper, WP/10/201.

[11] Harzi, A., (2011), « The impact of Basel III on Islamic banks: A theoretical study and comparison with conventional banks », Research Chair of Ethics and Financial Norms, Paris 1 Sorbonne and King Abdul Universities.

[12] Khan, F. (2010) How Islamic is Islamic banking? Journal of Economic Behavior \& Organization 76, 805-820.

[13] Kraisicka et Nowak (2012), «What's in it for me? A primary differences between Islamic and conventional banks », International Monetary Fund Working Paper, $\mathrm{WP} / 12 / 151$

[14] Metwally, M.M., (1997), « Differences between the financial characteristics of interest-free banks and conventional banks », European Business review 97, 2, 92-98. 
[15] Pappas, V., Izzeldin, M., et Fuertes, A., (2012), «Failure Risk in Islamic and Conventional banks », Lancaster University and City University, United Kingdom.

[16] Siddiqui, M.N., (2002), « Dialogue in Islamic Economics », Lahore/London, Institute of Policy Studies/The Islamic Foundation.

[17] Sundararajan, V., et Errico, L., (2002), « Islamic Financial Institutions and Products in the Global Financial System: Key Issues in Risk Management and Challenges Ahead », International Monetary Fund Working Paper, No. 02/192. 


\section{Tableaux partie empirique}

Tableau 4

Une comparaison des caractéristiques financières du modèle d'intermédiation des banques islamiques avec celui des banques conventionnelles

\begin{tabular}{|c|c|c|c|c|c|c|}
\hline & \multicolumn{2}{|c|}{$\begin{array}{l}\text { Ratios de fonds propres de } \\
\text { base }\end{array}$} & \multicolumn{2}{|c|}{$\begin{array}{l}\text { Ratios de fonds propres } \\
\text { complémentaires }\end{array}$} & \multicolumn{2}{|c|}{ Ratios de capital globaux } \\
\hline & $\mathrm{a}$ & $\mathrm{b}$ & $\mathrm{a}$ & $\mathrm{b}$ & $\mathrm{a}$ & $\mathrm{b}$ \\
\hline & T1RP & T1TAP & T2RP & T2TAP & TCRP & TCTAP \\
\hline \multirow[t]{2}{*}{ Banques islamiques } & $3.123 * *$ & $3.427 * * *$ & $-0.53 * * *$ & $-0.629 * * *$ & 2.389 & $2.615 *$ \\
\hline & $(1.4463)$ & (1.4578) & $(0.1922)$ & $(0.1726)$ & $(1.4601)$ & $(1.3569)$ \\
\hline \multirow[t]{2}{*}{ Taille } & $-2.498 * * *$ & $-2.118 * * *$ & $0.291 * * *$ & 0.012 & $-2.52 * * *$ & $-2.077 * * *$ \\
\hline & $(0.2632)$ & $(0.2241)$ & $(0.0453)$ & $(0.0489)$ & $(0.2538)$ & $(0.2161)$ \\
\hline \multirow{2}{*}{ Crédits/total actifs } & -0.064 & 0.009 & 0.002 & $0.014 * *$ & $-0.126 * * *$ & 0.011 \\
\hline & $(0.0417)$ & $(0.0396)$ & $(0.0052)$ & $(0.0377)$ & $(0.0335)$ & $(0.038)$ \\
\hline \multirow[t]{2}{*}{ OOIAAP } & $0.79 * *$ & $0 . .922 * * *$ & -0.004 & 0.000 & $0.549 *$ & $0.97 * * *$ \\
\hline & $(0.3232)$ & $(0.2551)$ & $(0.0353)$ & $(0.0377)$ & $(0.3035)$ & $(0.2419)$ \\
\hline \multirow[t]{2}{*}{ Actifs liquides/total actifs } & $0.094 * * *$ & 0.004 & -0.002 & 0.005 & $0.056^{* *}$ & 0.008 \\
\hline & $(0.0267)$ & $(0.0245)$ & $(0.005)$ & $(0.0053)$ & $(0.0236)$ & $(0.023)$ \\
\hline \multirow[t]{2}{*}{ Marge d'intérêt nette } & $0.37 * * *$ & $0.349 * * *$ & 0.039 & 0.039 & $0.476^{* * *}$ & $0.404 * * *$ \\
\hline & $(0.1401)$ & $(0.1396)$ & $(0.0424)$ & $(0.036)$ & $(0.1557)$ & $(0.1425)$ \\
\hline $\mathrm{N}$ & 2552 & 2580 & 2507 & 2514 & 3704 & 2779 \\
\hline Pays et années & Oui & Oui & Oui & Oui & Oui & Oui \\
\hline $\mathrm{R} 2$ & 0.3901 & 0.4606 & 0.2 & 0.1387 & 0.3092 & 0.4524 \\
\hline
\end{tabular}

Tableau 5

Une comparaison des caractéristiques financières du modèle d'intermédiation des banques islamiques avec celui des banques conventionnelles (classification par taille)

\begin{tabular}{|c|c|c|c|c|c|c|}
\hline & \multicolumn{2}{|c|}{$\begin{array}{l}\text { Ratios de fonds propres } \\
\text { de base }\end{array}$} & \multicolumn{2}{|c|}{$\begin{array}{l}\text { Ratios de fonds propres } \\
\text { complémentaires }\end{array}$} & \multicolumn{2}{|c|}{ Ratios de capital globaux } \\
\hline & $\mathrm{a}$ & $\mathrm{b}$ & $\mathrm{a}$ & $\mathrm{b}$ & $\mathrm{a}$ & $\mathrm{b}$ \\
\hline & T1RP & T1TAP & T2RP & T2TAP & TCRP & TCTAP \\
\hline Banques islamiques de petite taille & $\begin{array}{l}16.643 * * * \\
(5.5798)\end{array}$ & $\begin{array}{l}16.37 * * * \\
(5.2893)\end{array}$ & $\begin{array}{l}-1.047 * * * \\
(0.2596)\end{array}$ & $\begin{array}{l}-1.126 * * * \\
(0.3126)\end{array}$ & $\begin{array}{l}10.901 * * \\
(5.284)\end{array}$ & $\begin{array}{l}16.014 * * * \\
(5.1254)\end{array}$ \\
\hline Banques islamiques de taille moyenne & $\begin{array}{l}2.447 \\
(1.8716)\end{array}$ & $\begin{array}{l}2.936 \\
(2.2742)\end{array}$ & $\begin{array}{l}-0.739 * * * * \\
(0.2128)\end{array}$ & $\begin{array}{l}-\mathbf{0 . 6 5 8} * * * * \\
(0.2307)\end{array}$ & $\begin{array}{l}1.346 \\
(1.8368)\end{array}$ & $\begin{array}{l}1.692 \\
(2.0567)\end{array}$ \\
\hline Banques islamiques de grande taille & $\begin{array}{l}0.549 \\
(1.592)\end{array}$ & $\begin{array}{l}0.17 \\
(1.407)\end{array}$ & $\begin{array}{l}-0.113 \\
(0.3258)\end{array}$ & $\begin{array}{l}-0.451 \\
(0.2206)\end{array}$ & $\begin{array}{l}0.376 \\
(1.4961)\end{array}$ & $\begin{array}{l}-0.659 \\
(1.3892)\end{array}$ \\
\hline $\mathrm{N}$ & 2552 & 2580 & 2507 & 2514 & 3704 & 2779 \\
\hline Contrôle - banque & Oui & Oui & Oui & Oui & Oui & Oui \\
\hline Contrôle - Pays et années & Oui & Oui & Oui & Oui & Oui & Oui \\
\hline $\mathrm{R} 2$ & 0.4186 & 0.4606 & 0.2030 & 0.1387 & 0.3209 & 0.4933 \\
\hline
\end{tabular}

Tableau 6

Une comparaison des caractéristiques financières du modèle d'intermédiation des banques islamiques avec celui des banques conventionnelles (classification par région)

\begin{tabular}{|c|c|c|c|c|c|c|}
\hline & \multicolumn{2}{|c|}{$\begin{array}{l}\text { Ratios de fonds propres } \\
\text { de base }\end{array}$} & \multicolumn{2}{|c|}{$\begin{array}{l}\text { Ratios de fonds propres } \\
\text { complémentaires }\end{array}$} & \multicolumn{2}{|c|}{ Ratios de capital globaux } \\
\hline & $\mathrm{a}$ & $\mathrm{b}$ & $\mathrm{a}$ & $\mathrm{b}$ & $\mathrm{a}$ & $\mathrm{b}$ \\
\hline & T1RP & T1TAP & T2RP & T2TAP & TCRP & TCTAP \\
\hline Banques islamiques dans les pays & 5.375 & -1.615 & 0.029 & -0.041 & 1.014 & -1.75 \\
\hline MENA & $(4.089)$ & $(1.847)$ & $(0.2826)$ & $(0.1265)$ & $(3.8647)$ & $(1.5622)$ \\
\hline Banques islamiques dans les pays GCC & $\begin{array}{l}3.876 * * \\
(1.8098)\end{array}$ & $\begin{array}{l}5.639 * * * \\
(2.0932)\end{array}$ & $\begin{array}{l}-0.694 * * \\
(0.3109)\end{array}$ & $\begin{array}{l}-0.731 * * * \\
(0.249)\end{array}$ & $\begin{array}{l}2.694 \\
(1.8875)\end{array}$ & $\begin{array}{l}4.361 * * \\
(2.0917)\end{array}$ \\
\hline Banques islamiques dans les pays EU & $\begin{array}{l}1.278 \\
(3.8943)\end{array}$ & $\begin{array}{l}0.453 \\
(2.3599)\end{array}$ & $\begin{array}{l}-0.595 * \\
(0.3295)\end{array}$ & $\begin{array}{l}-1.315 * * * \\
(0.3608)\end{array}$ & $\begin{array}{l}1.961 \\
(2.5312)\end{array}$ & $\begin{array}{l}-0.74 \\
(1.6193)\end{array}$ \\
\hline Banques islamiques dans les pays SEA & $\begin{array}{l}3.182 \\
(4.9051)\end{array}$ & $\begin{array}{l}5.058 \\
(5.9232)\end{array}$ & $\begin{array}{l}-0.24 \\
(0.335)\end{array}$ & $\begin{array}{l}-0.173 \\
(0.4116)\end{array}$ & $\begin{array}{l}5.582 \\
(4.9239)\end{array}$ & $\begin{array}{l}6.636 \\
(5.6982)\end{array}$ \\
\hline Banques islamiques dans les pays SUB & $\begin{array}{l}-6.685 * * * \\
(1.3815)\end{array}$ & $\begin{array}{l}-1.713 \\
(1.0599)\end{array}$ & $\begin{array}{l}-1.185 * * * \\
(0.3928)\end{array}$ & $\begin{array}{l}-\mathbf{0 . 9 8 3} * * * \\
(0.237)\end{array}$ & $\begin{array}{l}-5.853 * * * \\
(2.2579)\end{array}$ & $\begin{array}{l}-2.511 * * \\
(0.2119)\end{array}$ \\
\hline $\mathrm{N}$ & 2552 & 2580 & 2507 & 2514 & 3704 & 2779 \\
\hline Contrôle - banque & Oui & Oui & Oui & Oui & Oui & Oui \\
\hline Contrôle - Pays et années & Oui & Oui & Oui & Oui & Oui & Oui \\
\hline $\mathrm{R} 2$ & 0.396 & 0.471 & 0.2022 & 0.1435 & 0.3092 & 0.4524 \\
\hline
\end{tabular}


Tableau 7

Une comparaison des caractéristiques financières du modèle d'intermédiation des banques islamiques avec celui des banques conventionnelles (classification par périodes d'avant, de pendant et d'après crise financière de 2007 - 2009)

\begin{tabular}{|c|c|c|c|c|c|c|}
\hline & \multicolumn{2}{|c|}{$\begin{array}{l}\text { Ratios de fonds } \\
\text { propres de base }\end{array}$} & \multicolumn{2}{|c|}{$\begin{array}{l}\text { Ratios de fonds propres } \\
\text { complémentaires }\end{array}$} & \multicolumn{2}{|c|}{$\begin{array}{l}\text { Ratios de capital } \\
\text { globaux }\end{array}$} \\
\hline & $\mathrm{a}$ & $\mathrm{b}$ & $\mathrm{a}$ & $\mathrm{b}$ & $\mathrm{a}$ & $\mathrm{b}$ \\
\hline & T1RP & T1TAP & T2RP & T2TAP & TCRP & TCTAP \\
\hline \multirow[t]{2}{*}{ Banques islamiques avant la crise } & 0.932 & 4.306 & $-0.744 * *$ & -0.476 & 0.864 & 3.577 \\
\hline & $(2.5401)$ & $(2.7979)$ & $(0.3212)$ & $(0.3898)$ & $(2.7378)$ & $(2.9142)$ \\
\hline \multirow[t]{2}{*}{ Banques islamiques durant la crise } & $4.941 * *$ & $5.362 * * *$ & $-0.713 * * *$ & $-0.764 * * *$ & $4.537 * *$ & $4.859 * *$ \\
\hline & (2.0279) & $(1.8489)$ & $(0.2269)$ & $(0.1988)$ & $(2.1204)$ & $(1.8962)$ \\
\hline \multirow[t]{2}{*}{ Banques islamiques après la crise } & $2.75^{*}$ & 2.259 & $-0.372 *$ & $-0.579 * * *$ & 1.826 & 1.368 \\
\hline & $(1.5845)$ & $(1.5348)$ & $(0.2161)$ & $(0.1766)$ & $(1.5526)$ & $(1.3886)$ \\
\hline $\mathrm{N}$ & 2552 & 2580 & 2507 & 2514 & 3704 & 2779 \\
\hline Contrôle - banque & Oui & Oui & Oui & Oui & Oui & Oui \\
\hline Contrôle - Pays et années & Oui & Oui & Oui & Oui & Oui & Oui \\
\hline $\mathrm{R} 2$ & 0.392 & 0.4636 & 0.2009 & 0.1391 & 0.3108 & 0.4559 \\
\hline
\end{tabular}




\section{NOTES}

${ }^{\mathrm{i}} \mathrm{L}$ 'annexe 1 présente les principaux organismes réglementaires des banques islamiques et les différentes catégories d'institutions financières islamiques.

ii Certains auteurs critiquent l'approche en termes d'intermédiation pour les banques islamiques. Aussi, distinguent-ils entre banque et Masrif (nomination arabe d'une banque). Dans cette étude, nous nous référons aux travaux empiriques d'Abedifar et al. (2013) et Beck et al. (2013) qui expliquent l'intermédiation des banques islamiques ainsi qu'aux travaux de Khan (2010) et Bitar et Madiès (2013) qui se penchent sur la raison d'être de ces banques.

iii La structure du Passif est appréhendée essentiellement par le ratio Moudharabah/total des dépôts (MOUD).

iv La structure de l'Actif est captée essentiellement par le ratio Mourabaha/total des opérations commerciales (MOUR).

${ }^{v}$ Les ratios de fonds propres réglementaires sont décomposés en deux sous-groupes : les ratios des fonds propres sur actifs pondérés par le risque (T1RP avec au numérateur uniquement les fonds propres de base et TCRP avec l'ensemble des fonds propres) et les ratios des fonds propres sur actifs non ajustés du risque (T1TAP pour les fonds propres de base et TCTAP pour la totalité des fonds propres).

${ }^{v i}$ À partir d'une régression linéaire multiple et d'une régression médiane (non tabulée), nous avons pu confirmer que les différents ratios de capital des banques islamiques ont une tendance décroissante (IBTREND) comparativement aux banques conventionnelles. Ainsi, nous avons trouvé que le recours excessif aux comptes d'investissements dégrade la capitalisation des banques islamiques.

${ }^{\text {vii }}$ CAP signifie : ratios de fonds propres de base, de fonds propres complémentaires (T2RP pour fonds propres complémentaires pondérés par le risque et T2TAP pour fonds propres complémentaires non pondérés par le risque) et de capital. CONTROLE inclut cinq variables de contrôle au niveau de la banque : le logarithme du total des actifs pour contrôler la taille de la banque, les crédits sur le total des actifs pour contrôler le risque de crédit, les autres revenus sur le total des actifs pour mesurer les revenus générés par d'autres activités comme les placements immobiliers, actifs liquides sur le total des actifs pour étudier la relation entre la liquidité et la capitalisation bancaire et finalement la marge d'intérêt nette pour examiner l'impact de la profitabilité.

viii Pour les ratios de fonds propres de base $:$ coeff. $=3.12, \mathrm{p}<5 \%$ pour T11P et coeff. $=3.43, \mathrm{p}<1 \%$ pour T1TAP. Pour les ratios de capital globaux : coeff. $=2.61, \mathrm{p}<10 \%$ pour TCTAP.

${ }^{\text {ix }}$ coeff. $=-0.53, \mathrm{p}<1 \%$ pour T2RP et coeff. $=-0.63, \mathrm{p}<1 \%$ pour T2TAP.

${ }^{\mathrm{x}}$ coeff. $=16.64, \mathrm{p}<1 \%$ pour T1RP et coeff. $=16.37, \mathrm{p}<1 \%$ pour T1TAP.

${ }^{x i}$ La zone Asie du Sud-Est (South East Asia, dénommée SEA) rassemble le Bangladesh, Brunei, l'Indonésie, la Malaisie, le Pakistan, les Philippines et Singapour ; le Moyen-Orient et l'Afrique du Nord (Middle East and North Africa, dénommée MENA) inclut les pays suivants : l'Algérie, l'Egypte, l'Iran, l'Iraq, la Jordanie, le Liban, le Palestine, la Syrie, la Tunisie et le Yémen ; les pays du conseil de coopération du Golfe (Gulf Cooperation Council ou GCC) comprennent l'Arabie Saoudite, le Qatar, le Kuwait, les Emirats Arabes Unis et Bahreïn ; l'Europe (Europe, EU) est représentée par l'Angleterre et la Turquie; l'Afrique subsaharienne (Sub-Saharan Africa ou SUB) est représentée par la Gambie, la Mauritanie, le Soudan et l'Afrique du Sud.

xii Un terme d'interaction entre la variable binaire des banques islamiques et la variable dédiée à chacune des régions en question; par exemple pour SEA : IBDV $\times$ SEA. 